\title{
Caracterización del trastorno por videojuegos: ¿Una problemática emergente?1
}

\author{
Eduardo Sandoval-Obando 2 \\ Universidad Autónoma de Chile (Chile)
}

Recibido: 09/05/2019 Aceptado: 02/06/2020

\begin{abstract}
Resumen
Objetivo. Sistematizar la literatura actual disponible en torno a la caracterización del trastorno por videojuegos (VDJ), aportando a la comprensión de este fenómeno en el contexto latinoamericano y su incidencia en la población infanto-juvenil. Método. Se utilizó una revisión sistemática de literatura, de corte analítico. Como eje de búsqueda se consideraron las categorías: (a) uso de las TIC, (b) videojuegos, (c) adicción y (d) trastorno por videojuegos, de acuerdo con estudios publicados en las bases de datos PubMed, ScienceDirect y Google Scholar, teniendo como referencia el DSM-V y CIE-11. Resultados. Las manifestaciones clínicas descritas para su uso adictivo son aún heterogéneas. También, se pudo observar que, cuanto más temprano se empieza, mayor será la frecuencia de su uso en edades posteriores, lo que aumenta las conductas de riesgo en el futuro y su incidencia en el tiempo de ocio y la recreación cotidiana. Conclusión. Los efectos que puede generar el uso problemático de dispositivos tecnológicos en el desarrollo de las personas, se contrapone al uso de los VDJ en el tratamiento de diversos trastornos como en el plano educativo. Adicionalmente, se reafirma el desafío de construir saberes e investigaciones multidisciplinares en torno al uso problemático de estos dispositivos.
\end{abstract}

Palabras claves. Videojuegos, adicción, salud mental, DSM-V, CIE-11.

\section{Characterization of Gaming Disorder: An Emerging Problem?}

\begin{abstract}
Objective. Systematize the current literature available on the characterization of video game disorder, in order to contribute to the understanding of this phenomenon in the Latin American context and its incidence in the child/youth population. Method. A systematic review and analysis of the literature was used. The following categories were considered lines of inquiry: (a) use of ICT, (b) video games, (c) addiction and (d) video game disorder, according to studies published in the PubMed, ScienceDirect and Google Scholar databases, having the DSM-V and ICD-11as references. Results. The clinical manifestations of its addictive use are still

Trabajo financiado por la Agencia Nacional de Investigación y Desarrollo (ANID) - FONDECYT de Iniciación N 11190028 “La Profesionalidad Docente Rural: Implicaciones Socioeducativas desde la Perspectiva Narrativa Generativa" (2019-2022).

2 Psicólogo, magíster en Educación y doctor en Ciencias Humanas. Académico adscrito a la Escuela de Psicología, Coordinador del Grupo de Investigación en Educación, Cultura y Transformación Social (ICET) e Investigador asociado al Instituto de Estudios Sociales y Humanísticos (IdeSH) - Facultad de Ciencias Sociales y Humanidades, Universidad Autónoma de Chile. Correo de correspondencia: eduardo.sandoval@uautonoma.cl Dirección postal: Avenida Alemania \# 01090 piso 6. Facultad de Ciencias Sociales y Humanidades, Universidad Autónoma de Chile - Temuco, Chile.
\end{abstract}


heterogeneous. Also, it was observed that the earlier it starts, the greater the frequency of its use in later ages, which increases risk behaviors in the future and its incidence in leisure time and daily recreation. Conclusion. The effects that the problematic use of technological devices can generate in the development of individuals are contrasted to the use of video games in the treatment of various disorders, such as in the educational field. Additionally, the challenge of building knowledge and multidisciplinary research around the problematic use of these devices is reaffirmed.

Keywords. Videogame, addiction, mental health, DSM-V, ICD-11.

\section{Caracterização do transtorno pelos videojogos: um problema emergente?}

\section{Resumo}

Escopo. Sistematizar a literatura atual disponível sobre a caracterização do transtorno pelos videojogos (VDJ), aportando à compreensão de este fenômeno no contexto latino-americano e sua incidência na população infanto-juvenil. Metodologia. Foi empregada uma revisão sistemática de literatura, de corte analítico. Como eixo de procura foram consideradas as categorias: (a) uso das TIC, (b) Videojogos, (c) Vício e (d) transtorno pelos videojogos, de acordo com estudos publicados nas bases de dados PubMed, ScienceDirect e Google Scholar, tendo como referencia o DSM-V e C1E-11. Resultados. As manifestações clínicas descritas para o seu uso aditivo ainda são heterogéneas. Também, foi observado que, quanto mais cedo começar, maior será a frequência do seu uso em idades posteriores, o que aumenta as condutas de risco no futuro e sua incidência no tempo de ócio e a recreação cotidiana. Conclusão. Os efeitos que pode gerar o uso problemático de dispositivos tecnológicos no desenvolvimento das pessoas, está contraposto ao uso dos VDJ no tratamento dos diversos transtornos como no plano educativo. Além do mais, é reafirmado o desafio de construir saberes e pesquisas multidisciplinares sobre o uso problemático de estes dispositivos.

Palavras-chave. Videojogos, adição, saúde mental, DSM-V, CIE-11.

\section{Introducción}

La evolución de las tecnologías de la información y la comunicación, así como la amplia cobertura y acceso a internet (Pinto y González, 2016), han incidido en la utilización creciente de videojuegos en la población infanto-juvenil, transformándolos en un problema de salud mental (Unicef, 2017).

En el contexto latinoamericano, Chile lidera el consumo de internet (Internet Media Services, 2016). Se estima que al menos el $85 \%$ de la población lo utiliza diariamente (Pinto y González, 2016). No obstante, pareciera ser que la revolución digital (Barriga, 2016; Dutta, Geiger y Lanvin, 2015) a la que se exponen tempranamente los niños, las niñas y los jóvenes (Palfrey y Gasser, 2008; Tapscott, 2011), instala problemáticas emergentes en el campo de la salud mental. Entre ellas, el uso problemático de los videojuegos (VDJ), descrito como un comportamiento adictivo de relevancia clínica aún controversial, tras su inclusión en el DSM-V (American Psychiatric Association, 2013) y CIE-11 (OMS, 2018). En este contexto, existen estudios que relacionan el uso problemático de los dispositivos tecnológicos con ciertos factores negativos para la salud de las personas, tales como el deterioro de las relaciones interpersonales, alteraciones del sueño y la alimentación (González-Bueso et al., 2018), la impulsividad (Gentile et al., 2011), el desarrollo de una baja autoestima (Lemmens, Valkenburg y Peter, 2011), el neuroticismo (Braun, Stopfer, Müller, Beutel y Egloff, 2016), el TDAH (VukosavljevicGvozden, Filipovic y Opacic, 2015), la ansiedad y la depresión (Bonnaire y Baptista, 2019).

De manera opuesta, Aarseth et al. (2017) y Lau, Stewart, Sarmiento, Saklofske y Tremblay (2018) señalan que los términos adicción a internet y uso patológico de internet se han utilizado de manera difusa, refiriéndose a todo tipo de actividades en las que se emplean las tecnologías de la información 
y la comunicación (TIC), entre ellas, los VDJ. Por lo tanto, es difícil distinguir con claridad el tipo de dispositivo utilizado, el medio en el que tiene lugar su uso (King y Delfabbro, 2013; Starcevic y Aboujaoude, 2017), así como las diferencias por sexo (González-Bueso et al., 2018). Por ende, resulta necesario preguntarse: ¿Cómo es caracterizado este trastorno en los manuales de clasificación diagnóstica?, ¿qué instrumentos se utilizan para su diagnóstico?, ¿qué impacto provoca el uso problemático de los VDJ en población infantojuvenil? Por consiguiente, el objetivo de este trabajo es sistematizar la literatura actual disponible en torno a la caracterización del trastorno por VDJ, en tanto se describen aquellos elementos que avalan o refutan su asociación con la adicción conductual, aportando a la comprensión y abordaje de este fenómeno en el contexto latinoamericano actual.

\section{Método}

En este trabajo se realizó una revisión sistemática de literatura (RSL), entendida como la búsqueda exhaustiva, objetiva y organizada en torno a los trabajos publicados más relevantes sobre un tema (Ramírez, 2007; Sáenz, 2001), procurando el análisis riguroso de la información (Onwuegbuzie y Frels, 2016). Para ello, se utilizó un diseño analítico, observacional y retrospectivo, adoptando como eje de búsqueda las siguientes categorías: (a) uso de las TIC, (b) videojuegos, (c) adicción y (d) trastorno por videojuegos. Este proceso está fundado en las directrices desarrolladas por Fink (1998), Maier (2013), Petticrew y Roberts (2008) y Sánchez-Meca (2010), así como en los criterios metodológicos de Ramírez y García-Peñalvo (2018).

Es importante señalar que para esta RSL se llevó a cabo una revisión, selección y análisis de los estudios publicados en las bases de datos PubMed, ScienceDirect y Google Scholar, ya que se trata de los catálogos de revistas mejor validados en el ámbito académico, por su rigor científico, calidad y factor de impacto e indexación (Dorta-González y Dorta-González, 2014; Howard, 2009).

\section{Criterios de inclusión y exclusión}

Para la búsqueda y selección de los estudios, se aplicaron los siguientes criterios de inclusión y exclusión (Codina, 2015):
- Son elegibles todas las publicaciones científicas que tengan directa relación con las categorías centrales escogidas uso de las TIC, videojuegos, adicción y trastorno por videojuegos-, a partir de la $\mathrm{RSL}$, revisiones sistemáticas o estudios de metaanálisis.

- Se analizaron estudios publicados preferentemente en idioma español o inglés, indexados en las bases de datos electrónicas PubMed, ScienceDirect y Google Scholar.

- Se priorizaron los hallazgos provenientes de la RSL, revisiones sistemáticas o metaanálisis, independientemente de su enfoque teórico o metodológico.

- Se excluyeron los artículos que no contaban con un diseño de investigación explícitamente definido, en lo relacionado con la formulación de hipótesis o preguntas de investigación, la definición del paradigma y el diseño del estudio, tales como workshops, encuestas, artículos de opinión, entre otros.

- Se utilizaron fuentes de información secundarias que contaran con una estructura rigurosa y precisa en su resumen. Es importante mencionar que no se consideraron revisiones terciarias, ni actualizaciones de revisiones.

\section{Procedimiento de búsqueda}

La búsqueda y selección de los estudios se desarrolló en mayo de 2019, y estuvo orientada por las categorías y criterios de inclusión y exclusión previamente mencionados. Las palabras clave se buscaron en el título y en el resumen de los trabajos analizados (Aguilar-Bustamante y CorreaChica, 2017). Inicialmente, la búsqueda electrónica arrojó un total de 4100 artículos relacionados con las palabras clave. No obstante, y tras el primer filtro de revisión y análisis teórico-metodológico, se seleccionaron 82 artículos, considerando su índice de impacto, así como el cumplimiento de los criterios de inclusión y exclusión debidamente indicados.

La información extraída fue contrastada con datos y fuentes oficiales aportados por organismos internacionales, tales como la Organización para la 
Colaboración y el Desarrollo Económicos (OCDE), la Organización Mundial de la Salud (OMS), la American Psychiatric Association (APA) y el Fondo de Naciones Unidas para la Infancia (Unicef).

\section{Estrategia de análisis de la información recopilada}

Tras la selección de las investigaciones, se procedió a su sistematización y contrastación semántica, en función de criterios temáticos y de repetición (Rodríguez, Lorenzo y Herrera, 2005). Este enfoque, de acuerdo con Sandoval-Obando (2017), facilita el análisis de cada estudio, a través de su representación temática, en donde las unidades de registro van siendo reducidas según el tema sobre el que tratan y la estructura o naturaleza de sus aportaciones, y de repetición, según la densidad e importancia que adquiere la categoría central en estudio, en función del propósito que persigue este trabajo.

\section{Muestra}

En este apartado se presenta una descripción global de las investigaciones revisadas, considerando cuatro indicadores que permiten contextualizar y caracterizar el tipo de muestra empleado, como resultado del proceso de la RSL. Específicamente, se tuvieron en cuenta el año de publicación, el tipo de indexación, las palabras clave y el idioma utilizado para la presentación del estudio (Tomàs, Feixas, Bernabeu-Tamayo y Ruiz, 2015). Estos datos fueron relevantes, puesto que con ellos fue posible hacer una descripción objetiva y rigurosa de los trabajos que conformaron esta revisión.

De los 82 estudios analizados, se encontró que la mayoría de ellos fueron publicados durante 2018 (18.3\%), lo cual evidencia el interés creciente del mundo científico por comprender en profundidad este fenómeno. De ahí que se convierta en un ámbito promisorio de estudio y desarrollo dentro de las ciencias sociales y de la salud (tabla 1).

Tabla 1

Clasificación de los estudios por año de publicación

\begin{tabular}{|c|c|c|c|c|c|c|c|c|c|c|c|}
\hline & \multicolumn{11}{|c|}{ Año publicación } \\
\hline & $\begin{array}{c}\text { Anterior a } \\
2009\end{array}$ & 2010 & 2011 & 2012 & 2013 & 2014 & 2015 & 2016 & 2017 & 2018 & 2019 \\
\hline $\begin{array}{l}\text { Núm. de } \\
\text { artículos }\end{array}$ & 11 & 7 & 6 & 4 & 9 & 5 & 9 & 5 & 5 & 15 & 6 \\
\hline$\%$ & 13.4 & 8.5 & 7.3 & 4.9 & 11 & 6.1 & 11 & 6.1 & 6.1 & 18.3 & 7.3 \\
\hline
\end{tabular}

Fuente: elaboración propia.

En cuanto a la indexación de los estudios revisados, fue relativamente homogénea según la base de datos en la que se encontraban: PubMed,

En lo relacionado con el idioma en que fueron publicadas las investigaciones analizadas, fue evidente la fuerte tendencia de la literatura anglosajona por el estudio, comprensión y abordaje de este fenómeno $(72 \%)$, contrastando con el
34 estudios (41.4\%); ScienceDirect, 24 estudios (29.3\%); y Google Scholar, 24 estudios (29.3\%) (tabla 2).

material disponible en idioma español (28\%) (tabla 2). Este hallazgo fue determinante para reafirmar la necesidad de explorar, sistematizar y profundizar en el estudio de este fenómeno en el contexto latinoamericano. 
Tabla 2

Clasificación de los estudios por idioma

\begin{tabular}{ccr}
\hline Idioma & Inglés & Español \\
\hline Núm. de artículos & 59 & 23 \\
$\%$ & 72 & 28 \\
\hline
\end{tabular}

Fuente: elaboración propia.

El análisis de los artículos revisados permitió identificar una estrecha relación entre las categorías claves empleadas -videojuegos, adicción y uso de las TIC- para esta RSL, lo que demostró una tendencia marcada a la caracterización del trastorno por videojuegos como una patología emergente en los manuales de clasificación diagnóstica actuales DSM-V y CIE-11 (tabla 3).

Tabla 3

Palabras con mayor frecuencia de repetición en estudios seleccionados

\begin{tabular}{cc}
\hline Palabras claves empleadas & Frecuencia \\
\hline Uso de las TIC & 35 \\
Videojuegos & 45 \\
Adicción & 42 \\
Trastorno por videojuegos & 27 \\
\hline
\end{tabular}

Fuente: elaboración propia.

\section{Resultados}

Las investigaciones que orientaron esta RSL, así como el análisis de cada artículo, permitieron responder, en parte, el propósito que perseguía este trabajo. Para facilitar su comprensión y lectura, a continuación, se presentan las principales temáticas, discusiones y reflexiones extraídas en categorías propositivas y emergentes, respecto a los principales asuntos que han orientado la discusión en torno a este fenómeno.

\section{De las TIC a los videojuegos: ¿Una realidad compleja y creciente?}

La incorporación progresiva de las TIC (GarcíaÁlvarez, 2013; Sandoval-Obando, 2018) y, particularmente, la intrusión de los videojuegos (VDJ), ha sido exponencial en los últimos años (Buiza-Aguado et al., 2017; Newzoo, 2018), explicado, en parte, por la cantidad de horas que pasan los niños, las niñas y los jóvenes frente a los dispositivos tecnológicos (Unicef, 2017), la exposición temprana a estas tecnologías (Lepicnik y Samec, 2013) y la débil supervisión con la que cuentan (Area, Gros y Marzal, 2008; MartínSantana, Fernández-Monroy y Galván-Sánchez, 2015). Asimismo, los dispositivos disponibles (consolas, computadores, teléfonos inteligentes, etc.) despiertan el interés de los usuarios ante la enorme variedad (Ameneiros y Ricoy, 2015; Yau, Crowley, Mayes y Potenza, 2012).

Por su parte, Guerra et al. (2019) señalan que la edad de inicio en el uso de internet es un factor relevante, puesto que cuanto más temprana sea su utilización, mayor será la frecuencia de su uso en edades posteriores (García, Portillo, Romo y Benito, 2007), lo que amplifica la emergencia de conductas de riesgo en el futuro (Montiel, 2014; Pérez, Hincapié y Arias-Cardona, 2018). A su vez, la encuesta de Bienestar Subjetivo en Estudiantes, realizada por la Organización para la Cooperación y el Desarrollo Económicos (OCDE, 2017) a alumnos de segundo medio de 72 países, devela que el $70 \%$ 
de los varones y el $27 \%$ de las mujeres mencionan el uso regular de videojuegos tras cumplir con su jornada escolar. Esto demuestra su incidencia en el tiempo de ocio y la recreación cotidiana.

No obstante, el uso de programas computarizados y VDJ no es totalmente negativo, puesto que ha mostrado un impacto positivo como estrategia de intervención ante patologías como TDAH (Ledo, De la Gándara, García y Gordo, 2016; Prins et al., 2013), autismo, depresión y ansiedad (Brito y Vicente, 2018), así como en la rehabilitación de pacientes con esquizofrenia mediante procesos de aprendizaje (Dorado y Castaño, 2018) que inciden en una mejora gradual del funcionamiento neurocognitivo y psicosocial de los pacientes. En cambio, Holtz y Appel (2011) hallaron que cuando el VDJ implica la interpretación de un personaje, lo que se denomina role-playing, se convierte en un elemento predictor de aislamiento y ansiedad en los más jóvenes (Van Patten, Weinstock y McGrath, 2018); lo cual devela las complejidades asociadas al uso de estas tecnologías en la revolución digital actual.

\section{Predilección de los videojuegos en la sociedad digital}

Los VDJ se dividen en pasivos o clásicos -que implican el uso de la coordinación visomotriz para interactuar con el dispositivo electrónico, demandando un bajo grado de actividad motrizy activos -aplicaciones que permiten al jugador utilizar parcial o completamente su cuerpo frente a los estímulos que aparecen en una pantalla(Moncada y Chacón, 2012). En los últimos, el éxito o fracaso en la partida depende de la destreza y movimiento frente a una cámara o dispositivo electrónico sensible a los movimientos (Foley y Maddison, 2010).

En el presente estudio se pudo observar literatura especializada que aborda los Massively Multiplayer Online Role-Playing Games (MMORPG). Según Carbonell (2014), en estas plataformas, el jugador se enfrenta a diferentes personajes y niveles de dificultad, y debe ejecutar diversas misiones, según el argumento central del VDJ, de ahí su carácter abierto. Los jugadores pueden conformar equipos y alianzas, además de construir rutas de desarrollo y progresión, puesto que el juego evoluciona y se transforma constantemente, independiente de que el jugador o equipo estén o no conectados u organizados entre sí (Ng y Wiemer-Hastings, 2005). Durante el desarrollo del juego, los participantes van adquiriendo premios e incentivos, lo que aumenta el interés y la dependencia por mantenerse vigentes en la partida.

De acuerdo con lo anterior, los MMORPG reúnen condiciones y criterios de funcionamiento que validan y normalizan comportamientos disfuncionales, tales como: (a) impunidad absoluta frente a las acciones que se realicen en el juego; (b) validación y normalización de las conductas de riesgo; (c) todas las conductas, ya sean buenas o malas, tienen recompensa, lo que tiene escasa lógica con la vida real actual (Di Blasi et al., 2019).

\section{Caracterización del uso problemático de los videojuegos}

La OMS ha venido recabando información clínica acerca del comportamiento adictivo a los VDJ. A partir de esta sistematización y el aún controversial debate (Aarseth et al., 2017; Lau et al., 2018), es incluido el trastorno por videojuegos (Gaming disorder) en el CIE-11 (OMS, 2018), y descrito como un patrón de juego continuo o recurrente, en el que se dan tres condiciones: (a) falta de control de la conducta de juego en cuanto al inicio, frecuencia, intensidad, duración y contexto en que se realiza; (b) priorización del juego frente a cualquier otro tipo de actividad; y (c) mantenimiento y escalada de la conducta, a pesar de tener conciencia de las consecuencias negativas que estos provocan. Esta constelación de criterios clínicos debe evidenciarse, al menos, durante un periodo de 12 meses.

Al respecto, Echeburúa y De Corral (2010) sugieren que el patrón adictivo de esta conducta no se debe tanto a la frecuencia con que se realiza, sino al tipo de relación que se establece con ella y al impacto negativo que ocasiona en los diferentes ámbitos de la vida (Davis, 2001). Es decir, los VDJ se convierten en una fuente diversa e intensa de gratificaciones que incitan su uso indiscriminado, puesto que se presentan en un formato digital innovador, dinámico y atractivo para los más jóvenes.

En este sentido, Fuster et al. (2012) plantean un modelo de cuatro motivaciones vinculadas al uso problemático de los VDJ en jóvenes: (a) socialización (mecanismo de vinculación a diversos grupos de pares), (b) exploración (interés por profundizar en el argumento central que propone el VDJ), (c) logro (deseo de alcanzar un mayor grado 
de validación, reconocimiento y liderazgo frente a otros jugadores), y (d) disociación (utilización del VDJ como fuente de evasión de la realidad). De esta manera, los autores identificaron cierta relación teórica entre socialización y exploración con el uso del VDJ adaptado, en contraste con la conexión entre logro y disociación como factor condicionante para la emergencia y desarrollo de un juego problemático o desadaptado.

\section{El trastorno por videojuegos: algunos instrumentos de valoración clínica}

La inclusión del trastorno por VDJ en el DSM-V (APA, 2013; Potenza, 2014) y CIE-11 (Aarseth et al., 2017; OMS, 2018), es un tema controversial y emergente, puesto que sus manifestaciones clínicas son descriptivas, heterogéneas y diversas (Gentile, Reimer, Nathanson, Walsh y Eisenmann, 2014). No obstante, según Buiza-Aguado et al. (2017), se han desarrollado instrumentos de medida que buscan identificar un patrón adictivo conductual en el uso de los VDJ. El primer instrumento desarrollado fue la Internet Addiction Scale, construida por Young (1998), quien más tarde desarrollaría la Video and Online Game Addiction (Flisher, 2010; Young, 2015). Posteriormente, Tejeiro y Bersabé (2002) diseñaron y validaron el cuestionario Problem Videogame Playing. De reciente desarrollo son la Gaming Addiction Scale (Lemmens, Valkenburg y Peter, 2009), la Video Game Addiction Test (Van Rooij, Schoenmakers, Van de Eijnden, y De Mheen, 2010) y la Internet Gaming Disorder Scale(Lemmens, Valkenburg y Gentile, 2015), que tiene estrecha conexión con los criterios descritos en el DSM-V. Este último consta de 21 ítems relacionados con los siete criterios de adicción, conflicto y síntomas de abstinencia, recaída y recuperación, prominencia cognitiva y de comportamiento, tolerancia y euforia. Además, evalúa el tiempo invertido por el sujeto al VDJ, la incapacidad para reducir su utilización y las consecuencias generadas en patrones de sueño y alimentación.

A pesar de esta diversidad de instrumentos, su validez psicométrica y aplicación en diferentes poblaciones y contextos, sigue siendo un tema pendiente (Paulus, Ohmann, Von Gontard y Popow, 2018). Por otro lado, King, Haagsma, Delfabbro, Gradisar y Griffiths (2013) mencionan ciertos patrones de comportamiento característicos en el trastorno por VDJ, entre los que destacan la abstinencia cuando no es posible jugar por internet, que incluye irritabilidad, ansiedad o tristeza, sin signos físicos de abstinencia farmacológica, falta de control y deterioro de las relaciones interpersonales.

\section{Discusión}

Para lograr el objetivo de este estudio de sistematizar la literatura actual disponible en torno a la caracterización del trastorno por VDJ, se consultaron investigaciones que muestran sus principales características. En primer lugar, fue posible confirmar la popularidad alcanzada por los dispositivos tecnológicos en niños, niñas (3-6 años) y jóvenes (International Telecommunication Union, 2017; Vandewater et al., 2007), independientemente de su estrato social (Lepicnik y Samec, 2013; Marengo, Herrera, Coutinho, Rotela y Strahler, 2015), puesto que se encuentran en un periodo sensible del desarrollo (Livingstone y Haddon, 2009). De hecho, la Unicef (2017) señala que son los jóvenes entre 15 y 24 años quienes más utilizan los dispositivos virtuales a través de internet $(71 \%)$, en comparación con el resto de la población (48\%).

Por otra parte, la distribución de usuarios por sexo es generalmente mayor en hombres ( $54 \%$ ) que en mujeres $(46 \%)$, de acuerdo con lo señalado en el informe de la Entertainment Software Association (2019). Esto es coherente con lo reportado a nivel mundial, ya que en 2017 los hombres utilizaron internet $12 \%$ más que las mujeres (Unicef, 2017). Por ello, precisamente, se ha observado que los hombres muestran una mayor prevalencia de conductas adictivas (5.8\%) que las mujeres (3\%), lo que implica que alcanzan una tasa más alta de adicción a los VDJ (Desai, Krishnan-Sarin, Cavallo y Potenza, 2010).

Más recientemente, Bonnaire y Baptista (2019) realizaron un estudio para explorar la relación entre el trastorno por VDJ con la depresión, la ansiedad y la alexitimia. Para ello, utilizaron una muestra de jóvenes $(n=429)$ conformada por 123 mujeres $(28.7 \%)$ y 306 hombres $(71.3 \%)$, con una edad media de 20.7 años y que participaban activamente en foros relacionados con VDJ. Así, hallaron que gran parte de los participantes (63.6\%) fueron identificados como jugadores problemáticos, entre ellos, nuevamente los hombres $(78.4 \%)$ puntuaron más alto que las mujeres (21.6\%). 
Desde el punto de vista demográfico, la RSL arrojó que los jugadores se distribuyen heterogéneamente en el mundo. Según lo reportado por Newzoo (2018), lidera Asia (36\%), seguida de América del Norte (32\%), Europa (28\%) y América Latina (4\%). No obstante, el liderazgo alcanzado por Asia en el consumo de VDJ estaría vinculado a su densidad poblacional y la tradición histórica del continente con el desarrollo y la producción de dispositivos tecnológicos en la economía mundial (Saadi, 2018).

En segundo lugar, el uso problemático de los VDJ puede convertirse en una actividad potencialmente dañina para la salud mental de niños, niñas y jóvenes (Chóliz y Marco, 2011), lo cual se evidencia en una muestra de jóvenes españoles, con 621 participantes, de los cuales $52.7, \%$ eran hombres y $47.3 \%$ eran mujeres. Los autores hallaron que los jóvenes entre 12 y 16 años juegan más días a la semana que los niños de 10 a 11 años, y le dedican mucho más tiempo a los juegos de consola. Asimismo, se ha observado que el uso reiterado de VDJ online, MMORPG, por ejemplo, eleva las posibilidades de socialización y normalización de conductas de riesgo en jóvenes situados en el rango de 16 a 21 años (Carbonell, 2014; Singh, 2019). En este ámbito, se ha encontrado cierta relación entre personas con TDAH y un mayor reporte de sintomatología adictiva en el uso de los VDJ. Es decir, se observó que el 23\% de las personas que utilizan estos dispositivos, reportan síntomas de adicción, independientemente del tipo de VDJ que prefieran (Mathews, Morrell y Molle, 2019). Este hallazgo muestra que ciertos grupos parecen estar en mayor riesgo de desarrollar adicción a los VDJ que otros, como los hombres, las personas más jóvenes, de 16 a 21 años, y las personas con TDAH (Mentzoni et al., 2011). A su vez, un estudio transversal de escolares estadounidenses $(n=4691$ niños(as) de $4^{\circ}$ y $5^{\circ}$ grado) encontró relación entre periodos de más de tres horas diarias de exposición a la televisión, el uso problemático de los VDJ y el consumo de alcohol y alucinógenos (Armstrong, Bush y Jones, 2010). Igualmente, se ha observado que el trastorno por VDJ está asociado con ciertos rasgos de personalidad disfuncionales, como la impulsividad (Gentile et al., 2011), la búsqueda de sensación (Mehroof y Griffiths, 2010), la baja autoestima (Lemmens et al., 2011), la timidez (Lau et al., 2018) y el neuroticismo (Braun et al., 2016). Por consiguiente, ante el escaso desarrollo de estudios prospectivos y longitudinales en esta materia, cobran relevancia los aportes de Gentile, Li, Khoo, Prot y Anderson (2014), quienes hallaron que la baja competencia social, la empatía disminuida y la pobre regulación emocional operan como factores de riesgo para el uso problemático de los VDJ, mientras que la depresión, la ansiedad, la fobia social y el bajo rendimiento académico emergen como sus consecuencias en niños, niñas y jóvenes.

En tercer lugar, los hallazgos de la RSL evidenciaron que existe cierto consenso a la hora de determinar la relación del uso problemático de los VDJ con las adicciones comportamentales. Específicamente, el uso de los VDJ por 8-10 horas o más por día, acumulando 30 horas o más por semana, sería la característica predominante en sujetos que desarrollan el trastorno por VDJ, con una mayor prevalencia en hombres dentro de un rango etario entre 12 y 20 años, aun cuando la incidencia de la edad en la prevalencia de este trastorno sigue siendo un aspecto controversial (APA, 2013; Paulus et al., 2018). Por ende, el comportamiento adictivo sería una conducta compulsiva y potencialmente dañina para la salud física y mental, que daría paso a la emergencia de un síndrome de abstinencia psicológico, en respuesta a la prohibición de esta conducta (Ferguson, Coulson y Barnett, 2011; Kaptsis, King, Delfabbro y Gradisar, 2016). A nivel experimental, se ha observado la activación de circuitos cerebrales específicos, sistema mesocorticolímbico, en jóvenes con trastorno por VDJ, similar a lo detectado en personas con adicción a las drogas (Hoeft, Watson, Kesler, Bettinger y Reiss, 2008). Sin embargo, la inclusión de este trastorno en el DSM-V y CIE-11 aún genera disonancia en algunos sectores del mundo sociosanitario. Al respecto, Aarseth et al. (2017) plantean que su integración podría tener implicancias adversas en la población, aumentando el riesgo del sobrediagnóstico, así como los casos de falsos positivos en niños y niñas. A pesar de este debate, es esperable que futuras investigaciones permitan precisar el curso y evolución del comportamiento adictivo a los VDJ, develando las consecuencias negativas que provocan en la salud del sujeto (Skoric, Teo y Neo, 2009; Stockdale y Coyne, 2018). De forma preliminar, se ha observado cierta relación entre la impulsividad y el funcionamiento cognitivo deficiente, ya que estas características hacen que sea más difícil para los 
sujetos desconectarse de los estímulos gratificantes que provocan los VDJ, aumentando así la búsqueda compulsiva de esta conducta (Ko et al., 2013).

Por último, se encontró que existe evidencia relacionada con ciertas características sociofamiliares, como un factor que incide en la utilización problemática de los VDJ en niños, niñas y jóvenes. Específicamente, estudios prospectivos muestran que un nivel socioeconómico bajo puede estar asociado a un mayor uso de VDJ (Pentz, SpruijtMetz, Chou y Riggs, 2011). Incluso, en aquellos hogares en los que ambos padres trabajan o existe una débil supervisión, se presenta predisposición al uso problemático de los VDJ en niños (Abedini, Zamani, Kheradmand y Rajabizadeh, 2012). Lo anterior también tiene relación con la disponibilidad de acceso y utilización indiscriminada de los dispositivos tecnológicos, cuando se encuentran insertos en los ambientes cotidianos del menor (Atkin, Corder y Van Sluijs, 2013).

\section{Conclusiones}

Al sistematizar aquellos elementos que respaldan y refutan la asociación controversial del trastorno por VDJ con la adicción conductual, descrita en los manuales de clasificación diagnóstica DSM-V y CIE-11, este trabajo señala los efectos y consecuencias que generaría el uso problemático de los dispositivos tecnológicos en el desarrollo de las personas. Asimismo, orienta la discusión al contexto local, en vista a que el consumo de estas tecnologías en la población infanto-juvenil chilena supera la media respecto a otros países de la región (Bringué y Sádaba, 2008). Es decir, esta es una generación precoz en el quehacer tecnológico (Aguaded, 2011), pues desde la edad preescolar conviven e interactúan con estos dispositivos sin una supervisión adecuada (Atkin at al., 2013; Lepicnik y Samec, 2013).

En otro ámbito, se señala que el uso de los VDJ ha mostrado un impacto positivo en diversas áreas, convirtiéndose en una herramienta complementaria efectiva de tratamiento frente al TDAH, el autismo, la depresión y la ansiedad en niños, niñas, jóvenes y adultos (Brito y Vicente, 2018; Ledo et al., 2016; Miltenberger y Charlop, 2015; Prins et al., 2013), la promoción de hábitos alimentarios saludables en niños y niñas (Hermans et al., 2018), así como la estimulación cognitiva en personas mayores (Glass, Maddox y Love, 2013; Lampit, Hallock y Valenzuela, 2014). Además, han incidido positivamente en la formación universitaria en carreras de la salud (Gentry et al., 2019; Turner, Johnston, Kebritchi, Evans y Heflich, 2018), la innovación pedagógica (De Freitas, 2018), el trabajo colaborativo y la resolución de problemas (Sung y Hwang, 2018), el uso lúdico de las TIC en la promoción del aprendizaje autorregulado (Valencia y Caicedo, 2017) y la estimulación de las funciones ejecutivas en estudiantes escolarizados situados en un rango etario entre 14 y 18 años, siempre y cuando el VDJ se diseñe rigurosamente con este propósito (Homer, Plass, Raffaele, Ober y Ali, 2018; Parrado, Muñoz y Henao, 2015).

En este sentido, se tensiona el rol que asumen los profesionales de la salud en la identificación de aquellas señales de alerta que manifiesten los niños, las niñas y los jóvenes como consecuencia del uso potencialmente problemático de los VDJ ante la inmadurez de la evidencia clínica disponible sobre este fenómeno. Por lo tanto, de acuerdo con Rojas (2008) y Aarseth et al. (2017), es conveniente: (a) regular el uso de estos dispositivos de manera proporcional a la etapa cronológica en la que se encuentra el individuo; $y$ (b) promover el uso seguro y responsable de internet, informándose de los contenidos observados por los niños, con el fin de contribuir a la regulación adecuada y democrática de estos dispositivos.

Frente a esto, conviene prestar atención a eventuales cambios de conducta que puedan manifestar los sujetos, cuando el uso de estas tecnologías se vuelve problemático. Estos cambios incluyen agresividad, irritabilidad, aislamiento, baja autoestima, comportamiento sexual precoz, así como rendimiento escolar disminuido, entre otros. Ante estas situaciones, es recomendable supervisar la interacción de los niños con estos dispositivos, para ayudarlos a que sean capaces de diferenciar entre el mundo real y el de fantasía, en particular cuando su contenido aborda temáticas relacionadas con la violencia, las conductas de riesgo y la competición (Singh, 2019). De hecho, el uso de los VDJ puede esconder otras problemáticas, que podrían estar afectando al sujeto en el plano interpersonal, emocional, cognitivo, familiar o social (Jubany, 2017; Martín-Fernández et al., 2017). Este tipo de problemáticas que se generan demandan 
el desarrollo de enfoques y estudios más amplios, complejos y coherentes con la transformación digital actual.

En síntesis, se concluye que es necesario desarrollar futuras investigaciones en el campo de las ciencias sociales y de la salud, en las que sea posible analizar y determinar en profundidad la relación entre los estilos de personalidad del jugador, el tipo y argumento central que se propone en el VDJ, las consecuencias negativas que se generan cuando su uso es problemático y el curso psicopatológico de este comportamiento (GonzálezBueso et al., 2018). De igual manera, es necesario alcanzar un mayor grado de consenso respecto a los criterios diagnósticos utilizados para el trastorno por VDJ, a través de la selección de los instrumentos psicométricos más idóneos para investigar esta problemática, así como la fenomenología clínica que sustenta su uso en el campo de la salud mental.

\section{Referencias}

Aarseth, E., Bean, A., Boonen, H., Colder, M., Coulson, M., Das, D.,... Van Rooij, A. (2017). Scholars' open Debate Paper on the World Health Organization ICD-11 Gaming Disorder proposal. Journal of Behavioral Addictions, 6(3). 267-270. doi: 10.1556/2006.5.2016.088

Abedini, Y., Zamani, B., Kheradmand, A., \& Rajabizadeh, G. (2012). Impacts of Mothers' occupation Status and Parenting Styles on Levels of Self-Control, Addiction to Computer Games, and Educational Progress of Adolescents. Addiction \& Health, 4(3-4), 102-110.

Aguaded, I. (2011). Niños y adolescentes: nuevas generaciones interactivas. Comunicar, 36(18), 7-8.

Aguilar-Bustamante, M. y Correa-Chica, A. (2017). Análisis de las variables asociadas al estudio del liderazgo: una revisión sistemática de la literatura. Universitas Psychologica, 16(1), 1-13. doi: 10.11144/Javeriana.upsy16-1 avae

Ameneiros, A. y Ricoy, M. (2015). Los videojuegos en la adolescencia: prácticas y polémicas asociadas. Revista de Estudios e Investigación en Psicología y Educación (13), 115-119. doi: 10.17979/reipe.2015.0.13.451
American Psychiatric Association [APA]. (2013). Section III. Emerging Measures and Models. Conditions for Further Study. Internet Gaming Disorder. In Diagnostic and Statistical Manual of Mental Disorders (5th ed.) (pp. 795-798). Arlington: Author.

Area, M., Gros, B. y Marzal, M. (2008). Alfabetizaciones y tecnologías de la información y la comunicación. Madrid: Síntesis.

Armstrong, K., Bush, H., \& Jones, J. (2010). Television and Video Game Viewing and its Association with Substance use by Kentucky Elementary School Students, 2006. Public Health Reports, 125(3), 433-440. doi: 10.1177/003335491012500312

Atkin, A., Corder, K., \&Van Sluijs, E. (2013). Bedroom Media, Sedentary Time and Screen-Time in Children: A Longitudinal Analysis. International Journal of Behavioral Nutrition and Physical Activity, 10(1), 137. doi: 10.1186/1479-586810-137

Barriga, M. (2016). Futuro presente: Cómo la nueva revolución digital afectará mi vida. Santiago de Chile: Cámara Chilena del Libro.

Bonnaire, C., \& Baptista, D. (2019). Internet Gaming Disorder in Male and Female Young Adults: The Role of Alexithymia, Depression, Anxiety and Gaming Type. Psychiatry Research, 272, 521530.

Braun, B., Stopfer, J., Müller, K., Beutel, M., \& Egloff, B. (2016). Comparing Regular Gamers, Non-Gamers, and Gaming Addicts and Differentiating between Game Genres. Computers in Human Behavior, 55, 406-412. doi: 10.1016/j.chb.2015.09.041

Bringué, X. y Sádaba, C. (Coords.) (2008). La generación interactiva en Iberoamérica 2008: Niños y adolescentes ante las pantallas. Barcelona: Ariel/Fundación Telefónica.

Brito, H. y Vicente, B. (2018). Realidad virtual y sus aplicaciones en trastornos mentales: una revisión. Revista Chilena de Neuro-psiquiatría, 56(2), 127-135. doi: 10.4067/s071792272018000200127

Buiza-Aguado, C., García-Calero, A., AlonsoCánovas, A., Ortiz-Soto, P., Guerrero-Díaz, M., 
González-Molinier, M. y Hernández-Medrano, I. (2017). Los videojuegos: una afición con implicaciones neuropsiquiátricas. Psicología Educativa, 23(2), 129-136. doi: 10.1016/j. pse.2017.05.001

Carbonell, X. (2014). La adicción a los videojuegos en el DSM-5. Adicciones, 26(2), 91-95. doi: 10.20882/adicciones.10

Chóliz, M. y Marco, C. (2011). Patrón de uso y dependencia de videojuegos en infancia $y$ adolescencia. Anales de Psicología, 27(2), 418426.

Codina, L. (2015). No lo Ilame análisis bibliográfico, Ilámelo revisión sistematizada. Y cómo llevarla a cabo con garantías: Systematized Reviews + SALSA Framework. Barcelona: Lluís Codina. Recuperado de https://www.lluiscodina.com/ revision-sistematica-salsa-framework/

Davis, R. (2001). A Cognitive-Behavioral Model of Pathological Internet Use. Computers in Human Behavior, 17(2), 187-195. doi: 10.1016/S07475632(00)00041-8

De Freitas, S. (2018). Are Games Effective Learning Tools? A Review of Educational Games. Journal of Educational Technology \& Society, 21(2), 7484.

Desai, R., Krishnan-Sarin, S., Cavallo, D., \& Potenza, M. (2010). Video-Gaming Among High School Students: Health Correlates, Gender Differences, and Problematic Gaming. Pediatrics, 126(6), 1414-1424. doi: 10.1542/ peds.2009-2706

Di Blasi, M., Giardina, A., Giordano, C., Lo Coco, G., Tosto, C., Billieux, J., \& Schimmenti, A. (2019). Problematic Video Game Use as an Emotional Coping Strategy: Evidence from a Sample of MMORPG Gamers. Journal of Behavioral Addictions, 8(1), 25-34. doi: 10.1556/2006.8.2019.02

Dorado, C. y Castaño, D. (2018). Efectividad de los programas computarizados en rehabilitación cognitiva de pacientes con esquizofrenia. Pensamiento Psicológico, 16(2), 73-86. doi: 10.11144/1714

Dorta-González, M. I. y Dorta-González, P. (2014). Factor de impacto agregado según campos científicos. Investigación Bibliotecológica:
Archivonomía, Bibliotecología e Información, 28(62), 15-28. doi: 10.1016/S0187-358X (14)72563-8

Dutta, S., Geiger, Th. y Lanvin, B. (2015). Informe global sobre tecnologías de la información 2015. Ginebra: Foro Mundial Económico.

Echeburúa, E. y De Corral, P. (2010). Adicción a las nuevas tecnologías y a las redes sociales en jóvenes: un nuevo reto. Adicciones, 22(2), 9195. doi: 10.20882/adicciones.196

Entertainment Software Association [ESA]. (2019). 2019 Essential Facts. About the Computer and Video Game Industry. Recovered from https://www.theesa.com/wp-content/ uploads/2019/05/2019-Essential-Facts-Aboutthe-Computer-and-Video-Game-Industry.pdf

Ferguson, C., Coulson, M., \& Barnett, J. (2011). A Metaanalysis of Pathological Gaming Prevalence and Comorbidity with Mental Health, Academic and Social Problems. Journal of Psychiatric Research, 45(12), 1573-1578. doi: 10.1016/j.jpsychires.2011.09.005

Fink, A. (1998). Conducting Literature Research Reviews: From Paper to the Internet. Thousand Oaks: Sage.

Flisher, G. (2010). Getting plugged in: An overview of Internet addiction. Journal of Paediatrics and Child Health, 46(10), 557-559. doi: 10.1111/j.1440-1754.2010.01879.x

Foley, L., \& Maddison, R. (2010). Use of Active Video Games to Increase Physical Activity in Children: A (Virtual) Reality? Pediatric Exercise Science, 22(1), 7-20. doi: 10.1123/pes.22.1.7

Fondo de las Naciones Unidas para la Infancia [Unicef]. (2017). Estado mundial de la infancia 2017: Niños en un mundo digital. New York: Autor. Recuperado de https://www.unicef.org/ spanish/sowc2017/

Fuster, H., Oberst, U., Griffiths, M., Carbonell, X., Chamarro, A., \& Talarn, A. (2012). Psychological Motivation in Online RolePlaying Games: A study of Spanish World of Warcraft Players. Anales de Psicología, 28(1), 274-280. doi: 10.6018/analesps

García-Álvarez, M. (2013). El rol de las tecnologías de la información y comunicación en la gestión del conocimiento: un desafío estratégico en 
el nuevo contexto empresarial. Revista de Ciencias Sociales (Ve), 19(2), 322-333.

García, F., Portillo, J., Romo, J. y Benito, M. (2007). Nativos digitales y modelos de aprendizaje. Comunicación presentada al IV Simposio Pluridisciplinar sobre Diseño, Evaluación y Desarrollo de Contenidos Educativos Reutilizables. Recuperado de http://ceur-ws. org/Vol-318/Garcia.pdf

Gentile, D., Choo, H., Liau, A., Sim, T., Li, D., Fung, D., \& Khoo, A. (2011). Pathological Video Game Use Among Youths: A Two-Year Longitudinal Study. Pediatrics, 127(2), e319-e329. doi: 10.1542/peds.2010-1353

Gentile, D., Li, D., Khoo, A., Prot, S., \& Anderson, C. (2014). Mediators and Moderators of Long-Term Effects of Violent Video Games on Aggressive Behavior: Practice, Thinking, and Action. JAMA Pediatrics, 168(5), 450-457. doi: 10.1001/jamapediatrics.2014.63

Gentile, D., Reimer, R., Nathanson, A., Walsh, D., \& Eisenmann, J. (2014). Protective Effects of Parental Monitoring of Children's Media Use: A Prospective Study. JAMA Pediatrics, 168(5), 479484. doi: 10.1001/jamapediatrics.2014.146

Gentry, S., Gauthier, A., L'Estrade, B., Wortley, D., Lilienthal, A., Tudor, L.,... Car, J. (2019). Serious Gaming and Gamification Education in Health Professions: Systematic Review. I Med Internet Res, 21(3), e12994. doi: 10.2196/12994

Glass, B., Maddox, W., \& Love, B. (2013). RealTime Strategy Game Training: Emergence of a Cognitive Flexibility Trait. PLOS ONE, 8(8), e70350. doi: 10.1371/journal.pone.0070350

González-Bueso, V., Santamaría, J., Fernández, D., Merino, L., Montero, E. y Ribas, J. (2018). Association between Internet Gaming Disorder or Pathological Video-Game Use and Comorbid Psychopathology: A Comprehensive Review. International Journal of Environmental Research Public Health, 15(4), 668. doi: 10.3390/ijerph15040668

Guerra, C., Montiel, I., Molina, N., Escalona, Y., Riquelme, J. y Rojas, L. (2019). Análisis de una Escala Breve de Conductas de Riesgo en Internet en jóvenes chilenos. Anuario de Psicología, 49, 33-39.
Hermans, R., Van den Broek, N., Nederkoorn, Ch., Otten, R., Ruiter, E., \& Johnson-Glenberg, M. (2018). Feed the Alien! The Effects of a Nutrition Instruction Game on Children's Nutritional Knowledge and Food Intake. Games for Health Journal, 7(3), 164-174. doi: 10.1089/ g4h.2017.0055

Hoeft, F., Watson, C., Kesler, S., Bettinger, K., \& Reiss, A. (2008). Gender Differences in the Mesocorticolimbic System During Computer Gameplay. Journal of Psychiatric Research, 42(4), 253-258. doi: 10.1016/j. jpsychires.2007.11.010

Holtz, P., \& Appel, M. (2011). Internet Use and Video Gaming Predict Problem Behavior in Early Adolescence. Journal of Adolescence, 34(1), 4958. doi: 10.1016/j.adolescence.2010.02.004

Homer, B., Plass, J., Raffaele, C., Ober, T., \& Ali, A. (2018). Improving High School Students' Executive Functions Through Digital Game Play. Computers \& Education, 117, 50-58. doi: 10.1016/j.compedu.2017.09.011

Howard, J. (2009). Humanities Journals Confront Identity Crisis. The Chronicle of Higher Education, 55(19), A1.

International Telecommunication Union [ITU]. (2017). Measuring the Information Society Report 2017 Volume 1. Geneva, Switzerland: Author. Recovered from https://www.itu.int/ en/ITU-D/Statistics/Documents/publications/ misr2017/MISR2017_Volume1.pdf

Internet Media Services [IMS]. (2016). IMS Mobile in Latam Study (2nd ed.). Miami, USA: Author. Recovered from https://www.imscorporate. com/news/Estudios-comScore/IMS-MobileStudy-Septiembre2016.pdf

Jubany, J. (2017). ¿Hiperconectados? Educarnos en un mundo digital. Barcelona: Lectio.

Kaptsis, D., King, D., Delfabbro, P., \& Gradisar, M. (2016). Withdrawal Symptoms in Internet Gaming Disorder: A Systematic Review. Clinical Psychology Review, 43, 58-66. doi: 10.1016/j. cpr.2015.11.006

King, D., \& Delfabbro, P. (2013). Issues for DSM-5: Video-Gaming Disorder? Australia, NZ. J. Psychiatry, 47(1), 20-22. doi: 10.1177/0004867412464065 
King, D., Haagsma, M., Delfabbro, P., Gradisar, M., \& Griffiths, M. (2013). Toward a Consensus Definition of Pathological Video-Gaming: A Systematic Review of Psychometric Assessment Tools. Clinical Psychology Review, 33(3), 331 342. doi: 10.1016/j.cpr.2013.01.002

Ko, C., Lui, G., Yen, J. Chen, J., Yen, C. \& Chen, C. (2013). Brain Correlates of Craving for Online Gaming Under Cue Exposure in Subjects with Internet Gaming Addiction and in Remitted Subjects. Addict Biology, 18(3), 559-569. doi: 10.1111/j.1369-1600.2011.00405.x

Lampit, A., Hallock, H., \& Valenzuela, M. (2014). Computerized Cognitive Training in Cognitively Healthy Older Adults: A Systematic Review and Meta-Analysis of Effect Modifiers. PLOS Medicine, 11(11), e1001756. doi: 10.1371/ journal.pmed.1001756

Lau, C., Stewart, S., Sarmiento, C., Saklofske, D., \& Tremblay, P. (2018). Who Is at Risk for Problematic Video Gaming? Risk Factors in Problematic Video Gaming in Clinically Referred Canadian Children and Adolescents. Multimodal Technologies and Interaction, 2(2), 19. doi: 10.3390/mti2020019

Ledo, A., De la Gándara, J., García, M. y Gordo, R. (2016). Videojuegos y salud mental de la adicción a la rehabilitación. Cuadernos de Medicina Psicosomática y Psiquiatría de Enlace (117), 72-83.

Lemmens, J., Valkenburg, P., \& Gentile, D. (2015). The Internet Gaming Disorder Scale. Psychological Assessment, 27(2), 567-582. doi: 10.1037/pas0000062

Lemmens, J., Valkenburg, P., \& Peter, J. (2009). Development and Validation of a Game Addiction Scale for Adolescents. Media Psychology, 12(1), 77-95. doi: 10.1080/15213260802669458

Lemmens, J., Valkenburg, P., \& Peter, J. (2011). Psychosocial Causes and Consequences of Pathological Gaming. Computers in Human Behavior, 27(1), 44-152. doi: https://doi. org/10.1016/j.chb.2010.07.015

Lepicnik, J. y Samec, P. (2013). Uso de tecnologías en el entorno familiar en niños de cuatro años de Eslovenia. Comunicar, 40(1), 119-126. doi: 10.3916/C40-2013-03-02
Livingstone, S. y Haddon, L. (2009). Introduction. En S. Livingstone y L. Haddon (Eds.), Kids Online: Opportunities and Risks for Children (pp. 1-6). Bristol: The Policy Press.

Maier, H. (2013). What Constitutes a Good Literature Review and Why Does its Quality Matter? Environmental Modelling \& Software, 43, 3-4. doi: 10.1016/j.envsoft.2013.02.004

Marengo, L., Herrera, L., Coutinho, T., Rotela, G. y Strahler, T. (2015). ¿Gamer o adicto? Revisión narrativa de los aspectos psicológicos de la adicción a los videojuegos. Neuropsicología Latinoamericana, 7(3), 1-12.

Martín-Santana, J., Fernández-Monroy, M. y GalvánSánchez, I. (2015). Valores y creencias de los jóvenes ante el policonsumo de sustancias adictivas. Revista de Ciencias Sociales (Ve), 21(4), 494-508.

Martín-Fernández, M., Matalí, J., García-Sánchez, S., Pardo, M., Lleras, M. y Castellano-Tejedor, C. (2017). Adolescentes con trastorno por juego en internet (IGD): perfiles y respuesta al tratamiento. Adicciones, 29(2), 125-133. doi: 10.20882/adicciones.890

Mathews, C., Morrell, H., \& Molle, J. (2019). Video Game Addiction, ADHD Symptomatology, and Video Game Reinforcement. The American Journal of Drug and Alcohol Abuse, 45(1), 6776. doi: 10.1080/00952990.2018.1472269

Mehroof, M., \& Griffiths, M. (2010). Online Gaming Addiction: The Role of Sensation Seeking, Self-Control, Neuroticism, Aggression, State Anxiety, and Trait Anxiety. CyberPsychol Behavior Social Network, 13(3), 313-316. doi: 10.1089/cpb.2009.0229

Mentzoni, R., Brunborg, G., Molde, H., Myrseth, H., Skouverøe, K., Hetland, J., \& Pallesen, S. (2011). Problematic Video Game Use: Estimated Prevalence and Associations with Mental and Physical Health. Cyberpsychology, Behavior, and Social Networking, 14(10), 591596. doi:10.1089/cyber.2010.0260

Miltenberger, C., \& Charlop, M. (2015). The Comparative Effectiveness of Portable Video Modeling Vs. Traditional Video Modeling Interventions with Children with Autism Spectrum Disorder. Journal of Developmental 
and Physical Disabilities, 27(3), 341-358. doi: 10.1007/s10882-014-9416-y

Moncada, J. y Chacón, Y. (2012). El efecto de los videojuegos en variables sociales, psicológicas y fisiológicas en niños y adolescentes. Retos. Nuevas tendencias en Educación Física, Deporte y Recreación (21), 43-49.

Montiel, I. (2014). Victimización juvenil sexual online: incidencia, características, gravedad y Co-ocurrencia con otras formas de victimización electrónica (Tesis de Doctorado en Psicología). Facultad de Psicología, Universidad de Valencia. Recuperado de http://roderic.uv.es/ handle/10550/43026

Newzoo. (2018). 2018 Global Games Market Report. Recovered from https://cdn2.hubspot. net/hubfs/700740/Reports/Newzoo_2018_ Global_Games_Market_Report_Light.pdf

$\mathrm{Ng}$, B., \& Wiemer-Hastings, P. (2005). Addiction to the Internet and Online Gaming. Cyberpsychology \& Behavior, 8(2), 110-3. doi: 10.1089/cpb.2005.8.110

Onwuegbuzie, A., \& Frels, R. (2016). 7 Steps to a Comprehensive Literature Review: A Multimodel \& Cultural Approach. London: Sage.

Organización para la Cooperación y el Desarrollo Económicos [OCDE]. (2017). PISA 2015 Results (Volume III): Students' Well-Being. Paris: OECD Publishing. Recovered from https://doi. org/10.1787/9789264273856-en

Organización Mundial de la Salud [OMS]. (2018). Mortality and Morbidity Statistics (CIE-11). Genève: Author. Recovered from https://icd. who.int/icd11 refguide/en/index.html

Palfrey, J., \& Gasser, U. (2008). Born Digital: Understanding the First Generation of Digital Natives. New York: Basic Books.

Parrado, F., Muñoz, J. y Henao, O. (2015). Diseño de videojuegos serios para la salud. Páginas: Revista Académica e Institucional de la UCPR, (95), 127-141. doi: 10.13140/ RG.2.1.2775.6965

Paulus, F., Ohmann, S., Von Gontard, A., \& Popow, C. (2018). Internet Gaming Disorder in Children and Adolescents: A Systematic Review.
Developmental Medicine \& Child Neurology, 60(7), 645-659. doi: 10.1111/dmcn.13754

Pentz, M., Spruijt-Metz, D., Chou, C., \& Riggs, N. (2011). High Calorie, Low Nutrient Food/ Beverage Intake and Video Gaming in Children as Potential Signals for Addictive Behavior. International Journal of Environmental Research and Public Health, 8(12), 4406-4424. doi: 10.3390/ijerph8124406

Pérez, M., Hincapié, B. y Arias-Cardona, A. (2018). Socialización de jóvenes a través de las TIC en una institución educativa de Antioquia. Pensamiento Psicológico, 16(2), 59-72. doi: 10.11144/1683

Petticrew, M., \& Roberts, H. (2008). Systematic Reviews in the Social Sciences: A Practical Guide. Indianapolis: John Wiley \& Sons.

Pinto, M. y González, B. (2016). Séptima Encuesta de Acceso, Usos y Usuarios de Internet. Santiago de Chile: Subsecretaría de Telecomunicaciones, Gobierno de Chile. Recuperado de http://www. subtel.gob.cl/wp-content/uploads/2015/04/ Informe-VII-Encuesta-de-Acceso-Usos-yUsuarios-de-Internet_VF.pdf

Potenza, M. (2014). Non-Substance Addictive Behaviors in the Context of DSM-5. Addictive Behaviors, 39(1), 1-2. doi: 10.1016/j. addbeh.2013.09.004

Prins, P. J., Ten Brink, E., Dovis, S., Ponsioen, A., Geurts, H., De Vries, M., \& Van der Oord, S. (2013). Braingame Brian: Toward an Executive Function Training Program with Game Elements for Children with ADHD and Cognitive Control Problems. Games for Health Journal, 2(1), 4449.

Ramírez, R. (2007). Calidad de vida relacionada con la salud como medida de resultados en salud: revisión sistemática de la literatura. Revista Colombiana de Cardiología, 14(4), 207-222.

Ramírez, M. y García-Peñalvo, F. (2018). Cocreación e innovación abierta: Revisión sistemática de literatura. Comunicar, 54, 9-18. doi: 10.3916/C54-2018-01

Rodríguez, C., Lorenzo, O. y Herrera, L. (2005). Teoría y práctica del análisis de datos cualitativos. Proceso general y criterios de 
calidad. Revista Internacional de Ciencias Sociales y Humanidades, 15(2). 133-154.

Rojas, V. (2008). Influencia de la televisión y videojuegos en el aprendizaje y conducta infanto-juvenil. Revista Chilena de Pediatría, 79(1), 81-85. doi: 10.4067/S037041062008000700012

Saadi, T. (2018). Revolución digital en Asia: Una nueva ola de innovación digital está reconfigurando Asia y elevando el potencial de crecimiento de la región. Finanzas y Desarrollo, 55(3), 31-33.

Sáenz, A. (2001). Leer e interpretar una revisión sistemática. Boletín de la Sociedad de Pediatría de Asturias, Cantabria, Castilla y León, 41(177), 215-221. Recuperado de https://www.sccalp. org/boletin/177/BolPediatr2001_41_215-221. pdf

Sánchez-Meca, J. (2010). Cómo realizar una revisión sistemática y un meta-análisis. Aula Abierta, 38(2), 53-64.

Sandoval-Obando, E. (2017). El docente como mediador emocional y cognitivo de jóvenes en contextos vulnerados: tensiones y desafíos para la transformación de la práctica pedagógica (Tesis de Doctorado en Ciencias Humanas). Universidad Austral de Chile. Recuperado de http://cybertesis.uach.cl/tesis/uach/2017/ egs218d/doc/egs218d.pdf

Sandoval-Obando, E. (2018). Aprendizaje e inteligencia artificial en la era digital: implicancias socio-pedagógicas ¿Reales o futuras? Revista Boletín Redipe, 7(11), 155-171.

Singh, M. (2019). Compulsive Digital Gaming: An Emerging Mental Health Disorder in Children. The Indian Journal of Pediatrics, 86(2), 171173. doi: 10.1007/s12098-018-2785-y

Skoric, M., Teo, L., \& Neo, R. (2009). Children and Video Games: Addiction, Engagement, and Scholastic Achievement. Cyberpsychol Behavior, 12(5), 567-572. doi: 10.1089/ cpb.2009.0079

Starcevic, V., \& Aboujaoude, E. (2017). Internet Addiction: Reappraisal of an Increasingly Inadequate Concept. CNS Spectr, 22(1), 7-13. doi: $10.1017 /$ S1092852915000863
Stockdale, L., \& Coyne, S. (2018). Video Game Addiction in Emerging Adulthood: CrossSectional Evidence of Pathology in Video Game Addicts as Compared to Matched Healthy Controls. J Affect Disord, 225(1), 265-272. doi: 10.1016/j.jad.2017.08.045

Sung, H., \& Hwang, G. (2018). Facilitating Effective Digital Game-Based Learning Behaviors and Learning Performances of Students Based on a Collaborative Knowledge Construction Strategy. Interactive Learning Environments, 26(1), 118134. doi: 10.1080/10494820.2017.1283334

Tapscott, D. (2011). Grown Up Digital: How the Net Generation Is Changing Your World. Nueva York: McGraw-Hill.

Tejeiro, R., \& Bersabé, R. (2002). Measuring Problem Video Game Playing in Adolescents. Addiction, 97(12), 1601-1606. doi: 10.1046/j.13600443.2002.00218.x

Tomàs, M., Feixas, M., Bernabeu-Tamayo, M. y Ruiz, J (2015). La literatura científica sobre rankings universitarios: una revisión sistemática. REDU Revista de Docencia Universitaria, 13(3), 33-54.

Turner, P., Johnston, E., Kebritchi, M., Evans, S., \& Heflich, D. (2018). Influence of Online Computer Games on the Academic Achievement of Nontraditional Undergraduate Students. Cogent Education, 5(1), 1-16. doi: 10.1080/2331186X.2018.1437671

Valencia, M. y Caicedo, A. (2017). Diseño de tareas apoyadas en TIC para promover aprendizaje autorregulado. Pensamiento Psicológico, 15(2), 15-28. doi: 10.11144/1586

Van Patten, R., Weinstock, J., \& McGrath, A. (2018). Health Outcomes in Individuals with Problem and Pathological Gambling: An Analysis of the 2014 North Carolina Behavioral Risk Factor Survey System (BRFSS). Journal of Gambling Studies, 34(1), 297-306. doi: 10.1007/s10899017-9712-4

Van Rooij, A., Schoenmakers, T., Van de Eijnden, R., \& De Mheen, D. (2010). Compulsive Internet Use: The Role of Online Gaming and Other Internet Applications. Journal of Adolescent Health, 47(1), 51-57. doi: 10.1016/j. jadohealth.2009.12.021 
Vandewater, E., Rideout, V., Wartella, E., Huang, X., Lee, J., \& Shim, M. (2007). Digital Childhood: Electronic Media and Technology Use Among Infants, Toddlers, and Preschoolers. Pediatrics, 119(5), e1006-e1015. doi: 10.1542/peds.20061804

Vukosavljevic-Gvozden, T., Filipovic, S., \& Opacic, G. (2015). The Mediating Role of Symptoms of Psychopathology Between Irrational Beliefs and Internet Gaming Addiction. Journal of RationalEmotive \& Cognitive-Behavior Therapy, 33(4), 387-405. doi: 10.1007/s10942-015-0218-7
Yau, Y., Crowley, M., Mayes, L., \& Potenza, M. (2012). Are Internet Use and Videogame Playing Addictive Behaviors? Biological, Clinical and Public Health Implications for Youths and Adults. Minerva Psichiatrica, 53(3), 153-170.

Young, K. (1998). Internet Addiction: The Emergence of a New Clinical Disorder. CyberPsychology and Behavior, 1(3), 237-244. doi: 10.1089/ cpb.1998.1.237

Young, K. (2015). Video and Online Game Addiction Test. Net Addiction. New York: NetAddiction. Recovered from http://netaddiction.com/areyou-an-obsessive-online-gamer/

Para citar este artículo / To cite this article / Para citar este artigo: Sandoval-Obando, E. (2020). Caracterización del trastorno por videojuegos: ¿Una problemática emergente? Pensamiento Psicológico, 18(1), 87-102. doi:10.11144/Javerianacali.PPSI18-1.ctvp 